\title{
Fast-track surgery protocol in elderly patients undergoing laparoscopic radical gastrectomy for gastric cancer: a randomized controlled trial
}

This article was published in the following Dove Press journal:

OncoTargets and Therapy

2 June 2016

Number of times this article has been viewed

\author{
Guozheng Liu' \\ Fengguo Jian ${ }^{2}$ \\ Xiuqin Wang ${ }^{2}$ \\ Lin Chen' \\ 'Department of General Surgery, \\ Chinese PLA General Hospital, \\ Beijing, People's Republic of China; \\ ${ }^{2}$ Second Department of General \\ Surgery, Changyi People's Hospital, \\ Shandong, People's Republic of China
}

Correspondence: Lin Chen Department of General Surgery, Chinese PLA General Hospital, No 28 Fuxing Road, Haidian District, Beijing 100853, People's Republic of China Tel +86 I38 01290395

Email chenlinpayi@।63.com
Aim: To study the efficacy of the fast-track surgery (FTS) program combined with laparoscopic radical gastrectomy for elderly gastric cancer (GC) patients.

Methods: Eighty-four elderly patients diagnosed with GC between September 2014 and August 2015 were recruited to participate in this study and were divided into four groups randomly based on the random number table as follows: FTS + laparoscopic group (Group A, $\mathrm{n}=21$ ), FTS + laparotomy group (Group B, n=21), conventional perioperative care $(\mathrm{CC})+$ laparoscopic group (Group C, $\mathrm{n}=21$ ), and $\mathrm{CC}+$ laparotomy group (Group D, n=21). Observation indicators include intrasurgery indicators, postoperative recovery indicators, nutritional status indicators, and systemic stress response indicators.

Results: Preoperative and intraoperative baseline characteristics showed no significant differences between patients in each group $(P>0.05)$. There were no significant differences between each group in nausea and vomiting, intestinal obstruction, urinary retention, incision infection, pulmonary infection, and urinary tract infection after operation $(P>0.05)$. Time of first flatus and postoperative hospital stay time of FTS Group A were the shortest, and total medical cost of this group was the lowest. For all groups, serum albumin, prealbumin, and transferrin significantly decreased, while CRP and interleukin 6 were significantly increased postoperative day 1 . From postoperative day 4-7, all indicators of the four groups gradually recovered, but compared with other three groups, those of Group A recovered fastest.

Conclusion: FTS combined with laparoscopic surgery can promote faster postoperative recovery, improve early postoperative nutritional status, and more effectively reduce postoperative stress reaction, and hence is safe and effective for elderly GC patients.

Keywords: Fast-track surgery program, postoperative nutritional status, postoperative recovery, postoperative stress reaction

\section{Introduction}

At present, the number of older people in People's Republic of China is increasing at a rapid rate. According to UN statistics, by 2050, nearly 500 million people will be over 60 years old in the People's Republic of China, while 2 billion people worldwide will be over the age of 60 . Because the incidence of gastric cancer (GC) increases with age, with the aging of the population, the number of elderly patients with GC is also increasing. Physiology of elderly GC patients declines, immunity reduces, tolerance to surgery is poor, and recovery is slow. Therefore, it is very important to find a more appropriate method of surgery and care for elderly GC patients.

Fast-track surgery (FTS) is defined as the integration of different medical intervention actively during perioperative period to accelerate the rehabilitation of patients 
undergoing operation. ${ }^{1}$ FTS programs, covering preoperative appropriate preparations and assessments, sophisticated operative manipulation, and standardized postoperative treatment and nurse care, have been proposed to maintain physiological function and thereby facilitate postoperative recovery. ${ }^{2}$ In recent years, FTS has been successfully applied to a variety of surgeries such as colorectal cancer, ${ }^{3}$ bladder cancer, ${ }^{4}$ esophageal cancer, ${ }^{5}$ and autologous breast reconstruction. ${ }^{6}$ Numerous studies have shown that FTS can improve the prognosis of patients, shorten the hospitalization time, and reduce complications. ${ }^{7}$

It has also been successfully applied in patients undergoing GC surgery. ${ }^{2,8-10}$ However, depending on the disease and patient population, the application of FTS is selective and has limitations. Several studies have reported that GC patients who accept FTS should be less than 80 years old. ${ }^{11-14}$ To date, there is scarce research on elderly patients (aged $\geq 75$ years) with GC.

Safety and efficacy of laparoscopic surgery for gastrointestinal diseases has also been confirmed by numerous studies. Zheng et $\mathrm{al}^{15}$ conducted a retrospective comparative study to show that laparoscopy-assisted distal gastrectomy was characterized by less intraoperative blood loss, less narcotic use, faster bowel function recovery, and shorter postoperative hospital stay compared with open distal gastrectomy for GC in elderly patients. However, it is controversial whether FTS combined with laparoscopic surgery has more benefits for GC in elderly patients. A study conducted by Abdikarim et $\mathrm{al}^{16}$ confirmed that the Enhanced Recovery After Surgery (ERAS) program combined with laparoscopy is associated with a shorter hospital stay in GC patients undergoing radical gastrectomy. However, some scholars believe that ERAS combined with laparoscopic surgery is not more beneficial for GC patients compared with either technique used alone. Therefore, we conducted a randomized controlled trial to compare postoperative outcomes among patients who received FTS proposal alone, those who received laparoscopic surgery alone, and those who receive FTS proposal combined with laparoscopic surgery to evaluate the clinical relevance of the FTS protocol and laparoscopic surgery in gastric surgery for elderly patients (60-80 years old) with GC.

\section{Patients and methods}

\section{Patients}

The study was approved by the Ethics Committee of Chinese PLA General Hospital, and written informed consent was obtained from the patients for the publication of this report and any accompanying images prior to the trial.
Patients were included based on inclusion and exclusion criteria. The inclusion criteria were as follows: a diagnosis of GC by a preoperative pathological biopsy using a gastroscope; age $\geq 60$ years; conforming with surgical indications and having no surgical contraindications according to "Japanese gastric cancer treatment statute"; and good compliance. The exclusion criteria were as follows: a history of cancer, abdominal surgery, and the presence of recent acute infection; tumor impregnated with serous or Stage IV according to intraoperative assessment; merging obstruction or perforation; receiving preoperative radiotherapy or chemotherapy; with contraindications of anesthesia or pneumoperitoneum; and autoimmune diseases, metabolic diseases, or major diseases of other systems.

Patients diagnosed with GC between September 2014 and August 2015 were recruited to participate in this study. Using PASS11 software, taking $\alpha=0.05$ (bilateral) and $\beta=0.10$, prealbumin as the main index and referring to the relevant literature, the estimated sample size was 84 cases. A total of 84 patients were divided into four groups randomly based on the random number table as follows: FTS + laparoscopic group (Group A, n=21), FTS + laparotomy group (Group $\mathrm{B}, \mathrm{n}=21$ ), conventional perioperative care $(\mathrm{CC})+$ laparoscopic group (Group $\mathrm{C}, \mathrm{n}=21$ ), and $\mathrm{CC}+$ laparotomy group (Group $\mathrm{D}, \mathrm{n}=21$ ).

\section{Operation and treatment}

All the operations and perioperative management were performed by the same experienced surgical team. FTS perioperative management was performed according to the "Gastrectomy Fast Track Surgery Guideline" formulated by 2014 European FTS Association. ${ }^{1}$ Table 1 lists the key elements of the FTS program in this study, which includes sufficient preoperative education, requires no preoperative bowel preparation and less transfusion, has shorter preoperative fasting time, intraoperative insulation, and smaller incisions, does not routinely use nasogastric tubes, provides good postoperative analgesia, and enables early ambulation and early oral feeding.

According to the National Comprehensive Cancer Network 2015 Clinical Practice Guidelines for GC, ${ }^{17}$ total gastrectomy with D2 lymph node dissection was used as the standard procedure. Depending on the location of the primary tumor, total, proximal subtotal, or distal subtotal gastrectomy was performed. All procedures were performed by the same team of surgeons. Intraoperative anesthesia consisted of a combination of epidural analgesia (Th7-11) and general anesthesia for patients in all groups. 
Table I Perioperative measures for FTS and conventional perioperative care

\begin{tabular}{|c|c|c|}
\hline Characters & FTS & CC \\
\hline \multicolumn{3}{|l|}{ Preoperative } \\
\hline Education & Gives great importance & Take no account \\
\hline Fasting & Fasting for $6 \mathrm{~h}$; water deprivation for $2 \mathrm{~h}$ & Fasting for $12 \mathrm{~h}$; water deprivation for $4 \mathrm{~h}$ \\
\hline Bowel preparation & No routine & Oral laxatives \\
\hline Gastric tube & $\begin{array}{l}\text { No routine or pull the gastric tube as soon as } \\
\text { possible after surgery }\end{array}$ & Preoperative routine use of nasogastric tube \\
\hline \multicolumn{3}{|l|}{ Intraoperative } \\
\hline $\begin{array}{l}\text { Transfusion and } \\
\text { insulation }\end{array}$ & $\begin{array}{l}\text { Intraoperative transfusion capacity is } 1,500 \mathrm{~mL} \\
\text { or less; intraoperative insulation routinely }\end{array}$ & $\begin{array}{l}\text { No routine intraoperative insulation; no control } \\
\text { of intraoperative transfusion capacity }\end{array}$ \\
\hline Incision processing & As small as possible & No special emphasis on creating a narrow incision \\
\hline \multicolumn{3}{|l|}{ Postoperative } \\
\hline Analgesia & $\begin{array}{l}\text { Nonsteroidal anti-inflammatory drug } \\
\text { intravenously after surgery twice daily }\end{array}$ & No anti-inflammatory drug given routinely \\
\hline Urine tube & Unplugged within $48 \mathrm{~h}$ & Unplugged 3-5 days after surgery \\
\hline Off-bed activity & One day after surgery & Do not require \\
\hline Diet & One day after surgery & After recovery of intestinal function \\
\hline
\end{tabular}

Abbreviations: FTS, fast-track surgery; CC, conventional perioperative care; $h$, hours.

Continuous thoracic epidural infusion of analgesics after surgery was administrated for 2 days for FTS groups and 3 days for CC groups. Nonsteroidal anti-inflammatory drugs were administrated intravenously after surgery twice daily for FTS groups, while no anti-inflammatory drugs were given routinely for CC groups. When the patient's temperature was normal, gastrointestinal function recovered, intravenous fluids were not required, and pain could be tolerated, so the patients were discharged. The discharged patients were followed for 30 days, and they could return to the hospital if they felt unwell. Patients who needed chemotherapy and/or radiotherapy were transferred to oncology for further treatment.

Observation indicators included intrasurgery indicators (including surgical approach, duration, amount of blood loss, and incision length), postoperative recovery indicators (including time of bowel function recovery, postoperative complications length of stay, and hospital costs), nutritional status indicators (including serum albumin, prealbumin, and transferrin before surgery and on the first, the fourth, and the seventh day after surgery), and systemic stress response indicators (including white blood cells, C-reactive protein [CRP], interleukin 6 [IL-6], and tumor necrosis factor $[\mathrm{TNF}]-\alpha$ before surgery and on the first, the fourth, and the seventh day after surgery).

\section{Data analysis}

Statistical Package for Social Scientists (SPSS, version 18.0, SPSS Inc., Chicago, IL, USA) was used for all analyses. The measurement data are represented as mean \pm standard deviation, while enumeration data are represented as percentages. Intergroup comparisons of measurement data were performed using variance analysis followed by LSD method, while intergroup differences were assessed using a $\chi^{2}$ test or Fisher's exact test for enumeration data.

\section{Results \\ Comparison of general preoperative information and intrasurgery indicators between treatment groups}

All 84 patients completed the treatment in this study. Preoperative and intraoperative baseline characteristics showed no significant differences between patients in each group, including age, sex, body mass index, tumor-node-metastasis (TNM) stage, complications, type of operation, operation time, and type of reconstruction ( $P>0.05$, Table 2$)$. Operation time was significantly short, blood loss during operation was significantly less, and incision length was significantly short in laparoscope groups (Groups A and C) than in laparotomy groups (Groups B and D; $P<0.05$, Table 2).

\section{Comparison of postoperative recovery indicators between treatment groups}

There were no significant differences between each group in nausea and vomiting, intestinal obstruction, urinary retention, incision infection, pulmonary infection, and urinary tract infection (all $P>0.05$ ). In laparoscopic groups, patients in Group A who received FTS had a shorter time of first flatus and postoperative hospital stay time compared with patients in Group C who received CC. In addition, total medical cost of Group A was significantly less than that of Group C. For laparotomy groups, time of first flatus and postoperative hospital stay time in Group B receiving FTS 
Table 2 Comparison of general preoperative information and intrasurgery indicators between treatment groups

\begin{tabular}{|c|c|c|c|c|c|c|}
\hline Characters & $\begin{array}{l}\text { Group A } \\
(n=2 I)\end{array}$ & $\begin{array}{l}\text { Group B } \\
(n=2 I)\end{array}$ & $\begin{array}{l}\text { Group C } \\
(n=2 I)\end{array}$ & $\begin{array}{l}\text { Group D } \\
(n=2 I)\end{array}$ & $\begin{array}{l}\chi^{2}(F) \\
\text { value }\end{array}$ & $P$-value \\
\hline Age (years, mean $\pm S D$ ) & $69.2 \pm 5.1$ & $67.8 \pm 3.9$ & $70.3 \pm 5.8$ & $68.6 \pm 4.9$ & $1.845^{*}$ & $>0.05$ \\
\hline $\operatorname{Sex}(n, M / F)$ & $10 / 11$ & $9 / 12$ & $12 / 9$ & $11 / 10$ & 0.952 & 0.81 \\
\hline \multicolumn{6}{|l|}{ Complication (n) } & $>0.05$ \\
\hline Cardiovascular system disease & 3 & 2 & 4 & 3 & 2.192 & 0.90 \\
\hline Respiratory system diseases & 2 & I & I & I & & \\
\hline Diabetes & I & 3 & 2 & 2 & & \\
\hline \multicolumn{7}{|l|}{ TNM staging } \\
\hline I & 2 & 3 & I & 3 & 1.841 & 0.93 \\
\hline ॥ & 10 & 9 & 9 & 10 & & \\
\hline III & 9 & 9 & II & 8 & & \\
\hline Time of operation (min, mean \pm SD) & $145 \pm 1 \mid .2$ & $150 \pm 13.9$ & $143 \pm 14.3$ & $155 \pm 12.8$ & $1.543 *$ & $>0.05$ \\
\hline \multicolumn{7}{|l|}{ Type of operation $(n)$} \\
\hline Distal gastrectomy & 12 & 10 & 9 & 10 & 1.169 & 0.98 \\
\hline Proximal gastrectomy & 4 & 5 & 6 & 6 & & \\
\hline Total gastrectomy & 5 & 6 & 6 & 5 & & \\
\hline \multicolumn{7}{|l|}{ Type of reconstruction (n) } \\
\hline Billroth-I & 6 & 5 & 6 & 7 & 6.56 & 0.89 \\
\hline Billroth-II & 9 & 10 & 8 & 9 & & \\
\hline Roux-en-Y & 6 & 4 & 7 & 3 & & \\
\hline Esophagogastrostomy & 0 & I & 0 & I & & \\
\hline Jejunal interposition & 0 & I & 0 & I & & \\
\hline Intraoperative blood loss ( $\mathrm{mL}$, mean $\pm \mathrm{SD}$ ) & $110 \pm 15.6$ & $175 \pm 18.8$ & $117 \pm 20.5$ & $165 \pm 19.6$ & $55.467 *$ & $<0.05$ \\
\hline Total incision length $(\mathrm{mm}$, mean $\pm \mathrm{SD})$ & $105 \pm 12.3$ & $210 \pm 18.5$ & $98 \pm 10.6$ & $198 \pm 15.6$ & $63.229 *$ & $<0.05$ \\
\hline
\end{tabular}

Notes: *F value; Group A, FTS + laparoscopic group; Group B, FTS + laparotomy group; Group C, conventional perioperative care + laparoscopic group; Group D, conventional perioperative care + laparotomy group.

Abbreviations: BMI, body mass index; FTS, fast-track surgery; min, minutes; TNM, tumor-node-metastasis; M, male; F, female.

were significantly shorter than Group D receiving $\mathrm{CC}$, and total medical cost of Group B was significantly less than that of Group D. The results are listed in Table 3.

\section{Comparison of nutritional status indicators and systemic stress response indicators between treatment groups}

Results of nutritional status indicators and systemic stress response indicators (Table 4) were as follows: On postoperative day 1 , serum albumin, prealbumin, and transferrin levels decreased significantly, while CRP and IL-6 levels increased significantly in all the four groups compared to preoperative value (all $P<0.05$ ). From postoperative day 4-7, all indicators of the four groups gradually recovered. Compared with the other three groups, all indicators in Group A patients recovered faster. On postoperative day 7 , serum albumin, prealbumin, and transferrin of Group A returned to the preoperative level, while those of the other three groups

Table 3 Comparison of postoperative recovery indicators between treatment groups

\begin{tabular}{|c|c|c|c|c|c|c|}
\hline Characters & $\begin{array}{l}\text { Group A } \\
(n=2 I)\end{array}$ & $\begin{array}{l}\text { Group B } \\
(n=21)\end{array}$ & $\begin{array}{l}\text { Group C } \\
(n=2 I)\end{array}$ & $\begin{array}{l}\text { Group D } \\
(n=2 I)\end{array}$ & $\begin{array}{l}\chi^{2}(F) \\
\text { value }\end{array}$ & $P$-value \\
\hline Time of first flatus (day, mean \pm SD) & $2.0 \pm 1.2$ & $3.1 \pm 1.0$ & $2.5 \pm 1.1$ & $3.6 \pm 0.9$ & $58.777^{*}$ & $<0.05$ \\
\hline Postoperative hospital stay time (day, mean \pm SD) & $6.3 \pm 1.5$ & $9.6 \pm 2.0$ & $7.8 \pm 1.8$ & $10.5 \pm 2.1$ & $54.15 *$ & $<0.05$ \\
\hline Total medical cost (thousand [RMB], mean $\pm S D$ ) & $33.6 \pm 2.8$ & $35.8 \pm 3.6$ & $38.7 \pm 1.9$ & $40.5 \pm 2.3$ & $32.86 *$ & $<0.05$ \\
\hline \multicolumn{7}{|l|}{ Postoperative complications } \\
\hline Nausea and vomiting & 2 & 3 & I & I & 1.7114 & $>0.05$ \\
\hline Gastric retention & 6 & 5 & I & 0 & 1.105 & $<0.05$ \\
\hline Intestinal obstruction & I & 0 & 0 & 0 & 3.036 & $>0.05$ \\
\hline Urinary retention & I & 2 & 2 & I & 0.718 & $>0.05$ \\
\hline Incision infection & 0 & I & 0 & 0 & 3.036 & $>0.05$ \\
\hline Pulmonary infection & 0 & 0 & 0 & I & 3.036 & $>0.05$ \\
\hline Urinary tract infection & I & 2 & 2 & 3 & 1.105 & $>0.05$ \\
\hline
\end{tabular}

Notes: *F value; Group A, FTS + laparoscopic group; Group B, FTS + laparotomy group; Group C, conventional perioperative care + laparoscopic group; and Group D, conventional perioperative care + laparotomy group.

Abbreviation: FTS, fast-track surgery. 
Table 4 Comparison of nutritional status indicators and systemic stress response indicators between treatment groups

\begin{tabular}{|c|c|c|c|c|}
\hline Characters & Before operation & I d after operation & $4 \mathrm{~d}$ after operation & $7 \mathrm{~d}$ after operation \\
\hline \multicolumn{5}{|c|}{ Serum albumin $(g / L)$} \\
\hline Group A & $31.5 \pm 3.2$ & $26.2 \pm 3.1^{*}$ & $32.5 \pm 2.4 * *$ & $38.7 \pm 4.8^{* *}$ \\
\hline Group B & $32.3 \pm 2.3$ & $27.1 \pm 2.2^{*}$ & $29.8 \pm 3.1$ & $33.9 \pm 3.6 * *$ \\
\hline Group C & $30.6 \pm 2.6$ & $26.1 \pm 1.8^{*}$ & $28.5 \pm 2.2$ & $30.7 \pm 1.9$ \\
\hline \multirow[t]{2}{*}{ Group D } & $31.7 \pm 2.1$ & $26.7 \pm 3.2^{*}$ & $27.8 \pm 3.3$ & $30.0 \pm 1.8$ \\
\hline & $F=3.26 ; P>0.05$ & $F=2.2 I ; P>0.05$ & $F=I .25 ; P>0.05$ & $F=56.23 ; P<0.05 * * *$ \\
\hline \multicolumn{5}{|c|}{ Prealbumin (mg/L) } \\
\hline Group A & $174.3 \pm 20.1$ & $132.3 \pm 15.2 *$ & $155.8 \pm 22.7 * *$ & $|78.5 \pm 2| .3 * *$ \\
\hline Group B & $176.2 \pm 19.8$ & $|3| .4 \pm 12.5 *$ & $133.5 \pm 2 \mid .5$ & $165.5 \pm 18.6 * *$ \\
\hline Group C & $175.1 \pm 20.2$ & $|34.2 \pm| 1.6 *$ & $135.7 \pm 22.3$ & $147.8 \pm 23.7$ \\
\hline \multirow[t]{2}{*}{ Group D } & $172.3 \pm 17.6$ & $133.5 \pm 16.3 *$ & $134.6 \pm 2 \mid .5$ & $145.3 \pm 22.9$ \\
\hline & $F=2.54 ; P>0.05$ & $F=3.77 ; P>0.05$ & $F=54.31 ; P<0.05 * * *$ & $F=63.5 \mathrm{I} ; P<0.05^{* * *}$ \\
\hline \multicolumn{5}{|c|}{ Transferrin (g/L) } \\
\hline Group A & $|.65 \pm 0.3|$ & $1.52 \pm 0.23$ & $1.60 \pm 0.22$ & $1.66 \pm 0.19$ \\
\hline Group B & $1.62 \pm 0.27$ & $1.48 \pm 0.14$ & $1.5 \mathrm{I} \pm 0.16$ & $1.58 \pm 0.25$ \\
\hline Group C & $|.63 \pm 0.2|$ & $1.39 \pm 0.18^{*}$ & $1.30 \pm 0.24^{*}$ & $1.38 \pm 0.37^{*}$ \\
\hline \multirow[t]{2}{*}{ Group D } & $1.66 \pm 0.35$ & $1.32 \pm 0.24^{*}$ & $1.21 \pm 0.15^{*}$ & $1.35 \pm 0.16^{*}$ \\
\hline & $F=2.66 ; P>0.05$ & $F=1.75 ; P>0.05$ & $F=2.35 ; P>0.05$ & $F=0.789 ; P>0.05$ \\
\hline \multicolumn{5}{|l|}{ CRP (mg/L) } \\
\hline Group A & $3.10 \pm 0.15$ & $35.21 \pm 15.62 *$ & $69.13 \pm|7.8|^{*}$ & $39.58 \pm 10.06$ \\
\hline Group B & $3.21 \pm 0.17$ & $36.83 \pm 17.36 *$ & $75.63 \pm 18.29 *$ & $49.41 \pm 13.05$ \\
\hline Group C & $3.03 \pm 0.21$ & $60.33 \pm 20.64 *$ & $90.13 \pm 22.28 * * * *$ & $68.07 \pm 12.34$ \\
\hline \multirow[t]{2}{*}{ Group D } & $3.42 \pm 0.35$ & $65.83 \pm 18.5 I^{*}$ & $95.13 \pm 27.82^{* * * *}$ & $78.21 \pm 21.13$ \\
\hline & $F=2.67 ; P>0.05$ & $F=64.63 ; P<0.05^{* * *}$ & $F=53.31 ; P<0.05^{* * *}$ & $F=44.65 ; P<0.05^{* * *}$ \\
\hline \multicolumn{5}{|l|}{ IL-6 (pg/mL) } \\
\hline Group A & $4 \pm 1$ & $82 \pm 15^{*}$ & $50 \pm 9 * *$ & $29 \pm 3 * *$ \\
\hline Group B & $3 \pm 1$ & $88 \pm 13^{*}$ & $55 \pm 10 * *$ & $35 \pm 6 * *$ \\
\hline Group C & $5 \pm 2$ & $180 \pm 23^{*}$ & $92 \pm 2 I^{* *}$ & $60 \pm 5 * *$ \\
\hline \multirow[t]{2}{*}{ Group D } & $5 \pm 1$ & $190 \pm 16 *$ & $98 \pm 24 * *$ & $68 \pm 6 * *$ \\
\hline & $F=4.89 ; P>0.05$ & $F=68.37 ; P<0.05^{* * *}$ & $F=52.90 ; P<0.05^{* * *}$ & $F=35.77 ; P<0.05^{* * *}$ \\
\hline
\end{tabular}

Notes: Group A, FTS + laparoscopic group; Group B, FTS + laparotomy group; Group C, conventional perioperative care + laparoscopic group; and Group D, conventional perioperative care + laparotomy group; $* P<0.05$ compared to preoperative value; $* * P<0.05$ compared to I $d$ after operation; $* * * P<0.05$ compared intergroup. Data are presented as mean \pm standard deviation.

Abbreviations: CRP, C-reactive protein; d, days; IL-6, interleukin 6; FTS, fast-track surgery.

were still significantly lower than the preoperative level. On postoperative day 7 , serum albumin and prealbumin were significantly higher in Group A than Groups B, C, and D $(P<0.05$; Group A $>$ Group B $>$ Group C $>$ Group D), while CRP and IL-6 were significantly lower than the other three groups $(P<0.05$, Group D $>$ Group C $>$ Group B $>$ Group A).

\section{Discussion}

Compared with younger people, the metabolic rate in elderly patients (60-80 years) is altered, cells undergo deformation and functional impairment, and major organs show reduced function or cell loss. They often experience systemic chronic diseases, such as cardiovascular system disease, respiratory system diseases, and diabetes, resulting in a decline in surgical tolerance and vital organ function, which easily leads to an underlying disease, chronic postoperative illness, or an acute attack. Therefore, the postoperative recovery for the elderly is a complex process, which should be given enough attention. For the elderly, the choice of surgical approach should be more careful. Surgical approach with less trauma and faster recovery is more appropriate.

FTS, first proposed by the Danish physician Kehlet, ${ }^{18}$ refers to the use of a series of perioperative management measures to reduce the patient's physical and psychological trauma stress to promote accelerated rehabilitation. Earlier studies have confirmed that FTS can reduce hospital stay, avoid or minimize short-term complications, and enable a speedier recovery and return to normal way of life, including eating a solid diet and earlier defecation time. ${ }^{19,20}$ In addition, Wang et $\mathrm{al}^{21}$ verified that ERAS can improve the stress reaction, decrease the patient's resting energy expenditure during the postoperative period, and hasten the rehabilitation of GC patients. Laparoscopy has rapidly developed in recent 10 years; and its curative effect on GC has been affirmed. ${ }^{22}$ Compared with celiotomy, the lesser 
body trauma and minimal effect on the nutritional status are beneficial to patient's postoperative recovery. Although FTS and laparoscopy have both been widely applied, there is scarce research on whether elderly GC patients receive benefits on combining FTS and laparoscope.

This randomized controlled trial was done to compare the effectiveness of FTS program vs conventional perioperative care in laparoscopic or open surgery for elderly GC patients. Our results showed that compared with CC, FTS resulted in a shorter time of first flatus and postoperative hospital stay time and less total medical cost, with no further complications occurring. As nutritional status indicators, the total change trends of serum albumin and prealbumin were consistent. Both serum albumin and prealbumin significantly decreased on postoperative day 1, and gradually returned to normal on postoperative day 4-7. Patients of FTS groups recovered faster, and the level of serum albumin and prealbumin in FTS groups on postoperative day 7 was even higher than that during preoperative level. Transferrin levels in FTS groups did not significantly reduce after surgery and were restored to preoperative levels on postoperative day 7 , while that of $\mathrm{CC}$ groups significantly declined after surgery and recovered slowly. Thus, gastrointestinal function and nutrition indicators of Group A patients who underwent FTS combined with laparoscopic surgery recovered fastest. There may be several reasons for this. First, laparoscopic surgery is considered a minimally invasive surgery. Compared with celiotomy, the lesser body trauma and minimal effect on the nutritional status are beneficial to patient's postoperative recovery.

However, opponents of laparoscopic surgery question the oncological quality of the procedure compared to the classical laparotomy approach. Comparison of laparoscopic and classical methods concluded that the number of lymph nodes removed during the operation is similar regardless of preferred technique. In addition, histopathological evaluation in all aforementioned operations proved both the laparoscopic surgery and classical laparotomy approach to be radical for GC patients. ${ }^{23,24}$ Second, early off-bed activity and diet can promote rapid recovery gastrointestinal function. Henriksen et $\mathrm{al}^{25}$ reported that early postoperative feeding helped to enhance anabolic and protect the intestinal mucosal barrier. Li et $\mathrm{al}^{26}$ confirmed that postoperative early enteral nutrition is safe, effective, produces functional recovery of the intestinal mucosa, and allows for a thorough recovery of patients with no increased complications. Third, patients in FTS groups accepted self-control analgesia, which led to good mental state and promoted postoperative rehabilitation.
When undergoing an operation, or because of other trauma, the body generates a stress response and produces a series of inflammatory mediators. CRP and IL- 6 are two of the important acute phase inflammatory reaction mediators. When the body suffers infection, trauma, or inflammation, serum concentrations of these mediators are significantly increased in 4-48 hours, and this increase is related to the trauma severity. Serum IL-6 concentration increase directly reflects the surgical trauma stimulation and damnification severity; among all cell factors, the relationship between IL-6 and conversion and prognosis is very close. ${ }^{27} \mathrm{CRP}$ can activate complement and strengthen the phagocytic role, thereby clearing the invading pathogens and cells with injury, apoptosis, and necrosis. They play an important protective role in the body's natural immune process. ${ }^{28}$ Our studies have shown that IL-6 is significantly increased postoperative day 1 . The increased level of IL-6 in the FTS groups (Groups A and B) is obviously lower than CC groups (Groups C and D), which is in accordance with the research of Hildebrandt et al. ${ }^{29}$ From postoperative day 4 to 7, IL-6 of all groups gradually decreased, but was still significantly higher than those of postoperative day 1. IL-6 of each group on postoperative day 7 was significantly different (Group D > Group C > Group B $>$ Group A). The total change trend of CRP is similar to that of IL-6. CRP of all groups significantly increased postoperative day 1 and gradually decreased from postoperative day 4 to 7. Moreover, CRP of CC groups (Groups C and D) declined more slowly than FTS groups (Groups A and B). This suggests that the operation can lead to obvious increase in IL-6 and CRP and damage the body; however, the degree of damage in the FTS groups was smaller than CC groups. The degree of the stress response in descending order is as follows: Group D > Group C > Group B > Group A. That is, from the perspective of the stress response, FTS combined with laparoscopic surgery is well tolerated by elderly GC patients.

This study has some limitations, such as selection bias and confounding bias. We tried to take measures to reduce bias as much as possible. First, we ensured that the experiment was randomized, controlled, and blinded correctly. In addition, surgery and perioperative management for all patients was done by the same group of doctors.

\section{Conclusion}

In conclusion, compared with CC and laparotomy, FTS combined with laparoscopic surgery can promote faster postoperative recovery, improve early postoperative nutritional status better, and more effectively reduce postoperative 
stress reaction, and hence is safe and effective for elderly GC patients.

\section{Acknowledgment}

We are grateful to the patients for participating in this study. This research received no specific grant from any funding agency in the public, commercial, or not-for-profit sectors.

\section{Disclosure}

The authors report no conflicts of interest in this work.

\section{References}

1. Bu J, Li N, Huang X, et al. Feasibility of fast-track surgery in elderly patients with gastric cancer. J Gastrointest Surg. 2015;19(8):1391-1398.

2. Chen ZX, Liu AH, Cen Y. Fast-track program vs traditional care in surgery for gastric cancer. World J Gastroenterol. 2014;20(2):578-583.

3. Bonde C, Khorasani H, Eriksen K, Wolthers M, Kehlet H, Elberg J. Introducing the fast track surgery principles can reduce length of stay after autologous breast reconstruction using free flaps: a case control study. J Plast Surg Hand Surg. 2015;49(6):367-371.

4. Taupyk Y, Cao X, Zhao Y, Wang C, Wang Q. Fast-track laparoscopic surgery: a better option for treating colorectal cancer than conventional laparoscopic surgery. Oncol Lett. 2015;10(1):443-448.

5. Guan X, Liu L, Lei X, et al. A comparative study of fast-track versus [corrected] conventional surgery in patients undergoing laparoscopic radical cystectomy and ileal conduit diversion: Chinese experience. $\mathrm{Sci}$ Rep. 2014;29(4):6820.

6. Zhao G, Cao S, Cui J. Fast-track surgery improves postoperative clinical recovery and reduces postoperative insulin resistance after esophagectomy for esophageal cancer. Support Care Cancer. 2014;22(2): 351-358.

7. Gustafsson UO, Scott MJ, Schwenk W, et al. Guidelines for perioperative care in elective colonic surgery: Enhanced Recovery After Surgery (ERAS $\left.{ }^{\circledR}\right)$ Society recommendations. Clin Nutr. 2012;31(6): 783-800.

8. Yamada T, Hayashi T, Cho H, et al. Usefulness of enhanced recovery after surgery protocol as compared with conventional perioperative care ingastric surgery. Gastric Cancer. 2012;15(1):34-41.

9. Pędziwiatr M, Matłok M, Kisialeuski M, et al. Enhanced recovery (ERAS) protocol in patients undergoing laparoscopic total gastrectomy. Wideochir Inne Tech Maloinwazyjne. 2014;9(2):252-257.

10. Beamish AJ, Chan DS, Blake PA, et al. Systematic review and metaanalysis of enhanced recovery programmes in gastric cancer surgery. Int J Surg. 2015;19:46-54.

11. Nanavati AJ, Prabhakar S. A comparative study of "fast-track" versus traditional peri-operative care protocols in gastrointestinal surgeries. J Gastrointest Surg. 2014;18(4):757-767.

12. Feng F, Ji G, Li JP, et al. Fast-track surgery could improve postoperative recovery in radical total gastrectomy patients. World J Gastroenterol. 2013;19:3642-3648.
13. Jeong O, Ryu SY, Jung MR, et al. The safety and feasibility of early postoperative oral nutrition on the first postoperative day after gastrectomy for gastric carcinoma. Gastric Cancer. 2014;17:324-331.

14. Hu JC, Jiang LX, Cai L, et al. Preliminary experience of fast-track surgery combined with laparoscopy-assisted radical distal gastrectomy for gastric cancer. J Gastrointest Surg. 2012;16:1830-1839.

15. Zheng L, Lu L, Jiang X, Jian W, Liu Z, Zhou D. Laparoscopy-assisted versus open distal gastrectomy for gastric cancer in elderly patients: a retrospective comparative study. Surg Endosc. 2015. Epub ahead of print.

16. Abdikarim I, Cao XY, Li SZ, Zhao YQ, Taupyk Y, Wang Q. Enhanced recovery after surgery with laparoscopic radical gastrectomy for stomach carcinomas. World J Gastroenterol. 2015;21(47):13339-13344.

17. Ajani JA, Barthel JS, Bekaii-Saab T, et al. Gastric cancer clinical practice guidelines in oncology (TM). J Natl Compr Canc Netw. 2010;8(4): 338-409.

18. Kehlet $\mathrm{H}$. Enhanced recovery after surgery (ERAS): good for now, but what about the future? Can J Anaesth. 2015;62(2):99-104.

19. Kehlet H, Kennedy RH. Laparoscopic colonic surgery - mission accomplished or work in progress? Colorectal Dis. 2006;8:514-517.

20. Scharfenberg M, Raue W, Junghans T, Schwenk W. "Fasttrack" rehabilitation after colonic surgery in elderly patients - is it feasible? Int $J$ Colorectal Dis. 2007;22:1469-1474.

21. Wang D, Kong Y, Zhong B, Zhou X, Zhou Y. Fast-track surgery improves postoperative recovery in patients with gastric cancer: a randomized comparison with conventional postoperative care. $J$ Gastrointest Surg. 2010;14:620-627.

22. Hu JH, Tang HN, Ma YP, et al. Systemic analysis on laparoscopeassisted gastrectomy for patients with gastric cancer. Asian Pac J Cancer Prev. 2015;16(5):2027-2029.

23. Cui M, Xing JD, Yang W, et al. D2 dissection in laparoscopic and open gastrectomy for gastric cancer. World J Gastroenterol. 2012;18: 833-839.

24. Wei HB, Wei B, Qi CL, et al. Laparoscopic versus open gastrectomy with D2 lymph node dissection for gastric cancer: a meta-analysis. Surg Laparosc Endosc Percutan Tech. 2011;21:383-390.

25. Henriksen MG, Hessov I, Dela F, Hansen HV, Haraldsted V, Rodt SA. Effects of preoperative oral carbohydrates and peptides on postoperative endocrine response, mobilization, nutrition and muscle function in abdominal surgery. Acta Anaesthesiol Scand. 2003;47(2):191-199.

26. Li B, Liu HY, Guo SH, et al. The postoperative clinical outcomes and safety of early enteral nutrition in operated gastric cancer patients. J BUON. 2015;20(2):468-472.

27. Seoane IV, Ortiz AM, Piris L, et al. Clinical relevance of VPAC1 receptor expression in early arthritis: association with IL-6 and disease activity. PLoS One. 2016;11(2):e0149141.

28. Jiao Y, Hu F, Zhang Z, et al. Effect of rosuvastatin dose-loading on serum sLox-1, hs-CRP, and postoperative prognosis in diabetic patients with acute coronary syndromes undergoing selected percutaneous coronary intervention (PCI). Int J Clin Exp Med. 2015;8(11):21565-21571.

29. Hildebrandt U, Kessler K, Plusczyk T, Pistorius G, Vollmar B, Menger MD. Comparison of surgical stress between laparoscopic and open colonic resections [J]. Surg Endosc. 2003;17:242-246.
OncoTargets and Therapy

\section{Publish your work in this journal}

OncoTargets and Therapy is an international, peer-reviewed, open access journal focusing on the pathological basis of all cancers, potential targets for therapy and treatment protocols employed to improve the management of cancer patients. The journal also focuses on the impact of management programs and new therapeutic agents and protocols on

\section{Dovepress}

patient perspectives such as quality of life, adherence and satisfaction. The manuscript management system is completely online and includes a very quick and fair peer-review system, which is all easy to use. Visit http://www.dovepress.com/testimonials.php to read real quotes from published authors. 